\title{
Development of Phase Contrast Scanning Transmission Electron Microscopy.
}

\author{
H. Iijima ${ }^{1}$, H. Minoda ${ }^{2,}$ T. Tamai ${ }^{2}$, Y. Kondo ${ }^{1}$, T. Fukuda and F. Hosokawa ${ }^{1}$. \\ ${ }^{1}$ EM Business Unit, JEOL Ltd., 3-1-2 Musashino, Akishima, Tokyo 196-8558, Japan. \\ ${ }^{2}$ Department of Applied Physics, Tokyo University of Agriculture and Technology, 2-24-16 Naka-cho, \\ Koganei, Tokyo 184-8588, Japan.
}

Phase contrast transmission electron microscopy (P-TEM) is a powerful tool to enhance the image contrast of transparent materials such as ice-embedded biological specimens or polymer materials. In PTEM, a phase plate is placed at the back-focal plane (BFP) of the objective lens (OL). It gives a phase shift for scattered electron waves, resulting in a change of phase contrast transfer function (PCTF) from sine to cosine type. Eventually, phase variation of specimens is converted into intensity variation. Among various types of phase plates, a carbon film phase plate with a small central hole is the most practical [1]. However, there is a serious issue that high-density electron beam (cross-over) on the phase plate causes the charging and/or the alteration of the phase plate, resulting in decreasing the life time of the phase plate.

To overcome this issue, we are developing phase contrast scanning transmission electron microscopy (PSTEM). Figure 1 shows the schematics of P-TEM and P-STEM. According to the reciprocity theorem, image contrast of P-STEM shows the same contrast in the P-TEM, if a phase plate is placed at a frontfocal plane (FFP) of an OL in P-STEM. In P-STEM, the life time of the phase plate is expected to improve, since a cross-over is not formed on the phase plate.

In our experiments, A field emission electron microscope (JEM-2100F) equipped with a Shottky electron source was used to obtain a coherent small probe on a specimen. Phase plate is placed on a condenser lens aperture plane that conjugates to the FFP of the OL.

Figure 2 compares conventional STEM (C-STEM) and P-STEM images of amorphous carbon film. A 30-nm-thick amorphous carbon film with a $5 \mu \mathrm{m}$-diameter central hole was used as phase plate. The power spectrum of Fourier transform for the P-STEM image shows that the PCTF changed from sine type to cosine type. It indicates that contrast converted image can be achieved in P-STEM as same as PTEM.

Figure 3 shows the long-life time of the phase plate in P-STEM. Figure 3 compares the Fourier transforms of P-STEM image with fresh phase plate (Fig. 3 (a)) and the plate after $100 \mathrm{~h}$ use (Fig. 3 (b)) using a sample of amorphous carbon film. It clearly shows that the phase plate remained stable after 100 hours. This result proves the life time of the phase plate is greatly improved by using P-STEM.

This development was supported by the program for "Development of Systems and Technologies for Advanced Measurement and Analysis" under JST.

\section{References:}

[1] R. Danev and K. Nagayama, J. Phys. Sci. Jpn. 70 (2001) p. 696. 


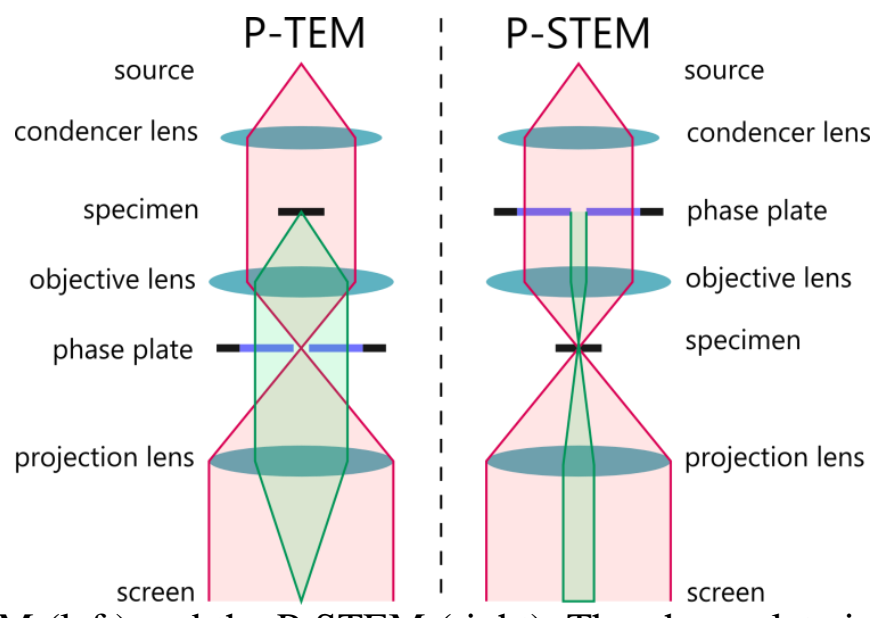

Fig. 1 Schematics of P-TEM (left) and the P-STEM (right). The phase plate is placed at the BFP of the objective lens in P-TEM and the FFP of objective lens in P-STEM.
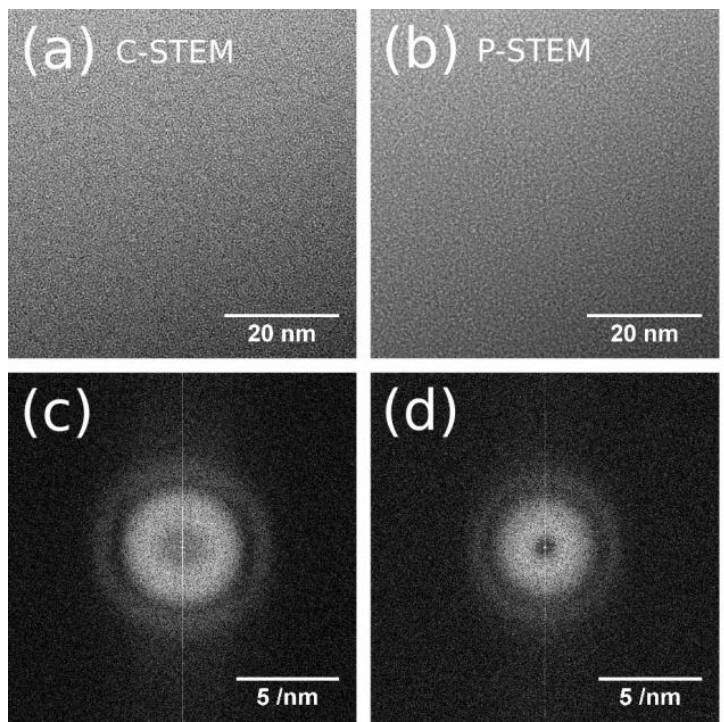

\section{(e)}

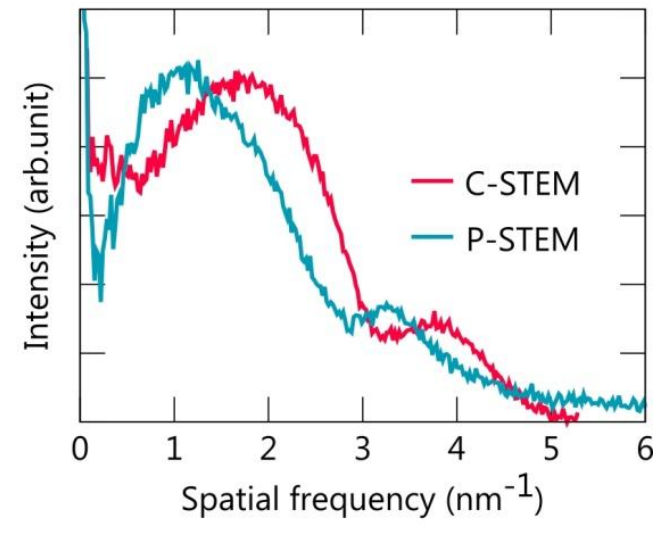

Fig. 2 C-STEM and P-STEM images of amorphous carbon film. Both images are taken at close to infocus. (a) C-STEM image. (b) P-STEM image. (c) and (d) Fourier transform of (a) and (b). (e) Intensity profiles of (c) and (d).
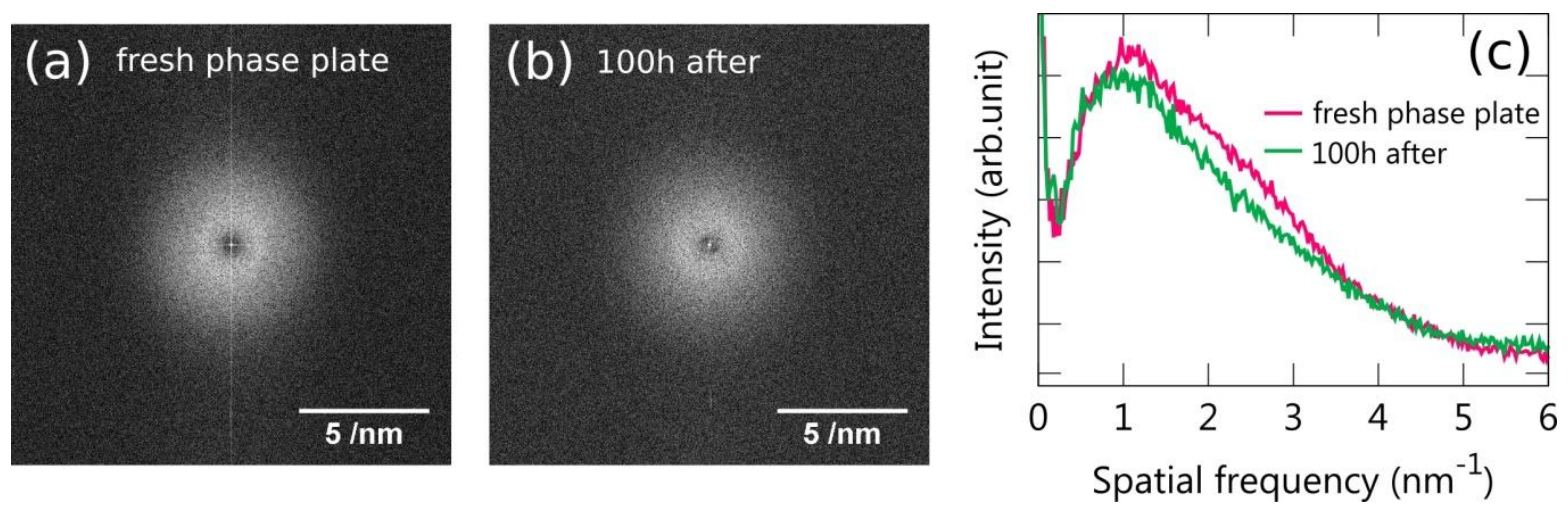

Fig. 3 Comparison of power spectrum of P-STEM images taken with fresh phase plate (a) and 100 hours used phase plate (b). (c) Intensity profiles of (a) and (b). 